\title{
Respiratory bronchiolitis-interstitial lung disease syndrome
}

INSERM

\section{Source}

INSERM. (1999). Orphanet: an online rare disease and orphan drug data base.

Respiratory bronchiolitis-interstitial lung disease syndrome. ORPHA:79127

Respiratory bronchiolitis - interstitial lung disease is a mild inflammatory pulmonary disorder developed by cigarette smokers and characterized by shortness of breath and cough, pulmonary function abnormalities of mixed restrictive and obstructive lung disease and high resolution CT scanning showing centrilobular micronodules, ground glass opacities and peribronchiolar thickening. 